\title{
OBSERVATIONS OF WATER AND MOLECULAR OXYGEN \\ IN THE INTERSTELLAR GAS
}

\author{
DAVID A. NEUFELD \\ The Johns Hopkins University, Baltimore, MD 21218
}

\begin{abstract}
Water and molecular oxygen are two simple molecules which are not easily observed in the interstellar medium. $\mathrm{O}_{2}$ emissions have yet to be detected from interstellar space, and $\mathrm{H}_{2} \mathrm{O}$ has been observed only in rather special environments where the interstellar gas has been warmed by embedded infrared sources or by shock waves. This sorry state of affairs does not necessarily reflect a low interstellar abundance for these species, but rather is a consequence of the very high abundance of $\mathrm{O}_{2}$ and $\mathrm{H}_{2} \mathrm{O}$ within our own atmosphere: strong atmospheric absorption lines make it extremely difficult to carry out observations of water and molecular oxygen emissions using ground-based or even airborne telescopes. Future observations from two orbiting telescopes scheduled for launch in the coming five years - the Infrared Space Observatory (ISO) and the Submillimeter Wave Astronomy Satellite (SWAS) - promise to improve radically the observational data on $\mathrm{H}_{2} \mathrm{O}$ and $\mathrm{O}_{2}$.
\end{abstract}

\section{Introduction}

Out of all the hundreds of interstellar molecules that have been detected or are potentially detectable, water and molecular oxygen are of particular interest for at least two reasons. First, the abundances of these species are of intrinsic interest in constraining our theories for the formation and destruction of interstellar molecules; simple species bearing the most abundant heavy element (oxygen) seem a good place to start in testing astrochemical models. Second, these molecules are potentially important coolants of molecular gas in the interstellar medium, both because they are potentially abundant, and - in the case of water - because of a large dipole moment. Indeed, in cold interstellar gas of density greater than $\sim 10^{6} \mathrm{~cm}^{-3}$, water may well be the dominant coolant (Goldsmith and Langer 1978) and thus may control the thermal balance and equilibrium temperature within cold dense cloud cores. Since gravitational collapse can occur only insofar as cold clouds are capable of cooling radiatively, the interstellar water abundance may prove relevant to the process of star-formation itself.

In this paper, I will review briefly attempts to observe $\mathrm{H}_{2} \mathrm{O}$ and $\mathrm{O}_{2}$ emission from two specific astrophysical environments: cold molecular clouds (with kinetic temperatures, $T \sim 10-40 \mathrm{~K}$ ); and hot shock-heated regions $\left(T \sim\right.$ few $\times 100-$ few $\left.\times 10^{3} \mathrm{~K}\right)$. Warm $(T \sim 100 \mathrm{~K})$ water has been extensively observed in radiatively-heated regions - such as the Orion hot core - 
which surround embedded infrared sources, but observations of $\mathrm{H}_{2} \mathrm{O}$ and $\mathrm{O}_{2}$ in this third astrophysical environment will not be considered here.

\section{Cold Dense Clouds}

\subsection{Observational UpPer Limits}

Neither water nor molecular oxygen has yet been observed from the cold quiescent gas which makes up much of the molecular material within the interstellar medium. Atmospheric absorption presents a tremendous problem for observations made from suborbital platforms. In the case of $\mathrm{O}_{2}$, two approaches have been adopted in attempting to detect emission from the interstellar gas. The first approach, suggested by Black and Smith (1984), is to search for radio emission from the isotopically substituted species ${ }^{16} \mathrm{O}^{18} \mathrm{O}$, for which the atmospheric attenuation is less severe. The theoretical calculations of Langer et al. (1984) suggest that no significant chemical fractionation is expected so that ${ }^{16} \mathrm{O}^{18} \mathrm{O} / \mathrm{O}_{2}=\left[{ }^{18} \mathrm{O}\right] /\left[{ }^{16} \mathrm{O}\right] \simeq 1 / 500$. Observations of six clouds by Goldsmith et al. (1985) have yielded (1 $\sigma)$ upper limits on the $\mathrm{O}_{2} / \mathrm{CO}$ abundance ratio in the range $0.5-4$. Listz and vandenBout (1985) have obtained corresponding upper limits $(1 \sigma)$ of 0.34 for Orion A and 0.07 for $\rho$ Ophiucus A. An alternative approach has been to search for $\mathrm{O}_{2}$ emission in external galaxies of sufficient redshift that the astrophysical $\mathrm{O}_{2}$ line emission would be shifted out of the corresponding atmospheric absorption feature; for the $(N, J)=(1,1)-(1,0)$ line at $119 \mathrm{GHz}$ the required redshift is $\sim 0.02$. This method has led to $(1 \sigma)$ upper limits on the (galaxyaveraged) $\mathrm{O}_{2} / \mathrm{H}_{2}$ abundance ratio of $1.4 \times 10^{-5}$ for NGC 7674 (Liszt 1985) and $4 \times 10^{-6}$ for VIIZw31 (Goldsmith and Young 1989).

In the case of $\mathrm{H}_{2} \mathrm{O}$, an upper limit upon the abundance within cold quiescent clouds has also been obtained by means of a search for the isotopically substituted $\mathrm{H}_{2}^{18} \mathrm{O}$ molecule. Observations of a set of seven clouds (which included both warm and cold regions) has been carried out from the Kuiper

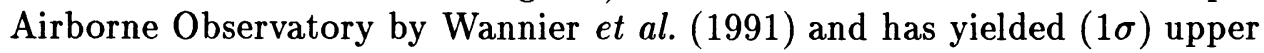
limits on the $\mathrm{H}_{2} \mathrm{O} / \mathrm{CO}$ abundance ratio in the range 0.001-0.03.

\subsection{Future Prospects}

The best upper limits on the $\mathrm{O}_{2}$ abundance are just beginning to become uncomfortable for theoretical models for the chemistry of oxygen-bearing species (e.g. Sternberg, Lepp, and Dalgarno 1987; Langer and Graedel 1989; Herbst and Leung 1989). Unfortunately, no significant improvements in sensitivity can be expected for observations carried out from within the Earth's atmosphere, and the full range of chemical model predictions will be tested only from space. The Submillimeter Wave Astronomy Satellite (SWAS) - 
a small Explorer mission to be launched by NASA in 1995 - has been designed to carry out a large scale survey of radio emission from five species of crucial importance to the oxygen chemistry (Melnick et al. 1991). The emission lines to be observed are: the $1_{10}-1_{01}$ line of $\mathrm{H}_{2} \mathrm{O}$ at $557 \mathrm{GHz}$; the corresponding line of $\mathrm{H}_{2}^{18} \mathrm{O}$ at $548 \mathrm{GHz}$; the $(N, J)=(3,3)-(1,2)$ line of $\mathrm{O}_{2}$ at $487 \mathrm{GHz}$; the $\mathrm{CI}^{3} \mathrm{P}_{1}-{ }^{3} \mathrm{P}_{2}$ fine-structure line at $492 \mathrm{GHz}$; and the ${ }^{13} \mathrm{CO} J=5-4$ line at $551 \mathrm{GHz}$. Vast improvements can be expected in the sensitivity to interstellar $\mathrm{O}_{2}$ and $\mathrm{H}_{2} \mathrm{O}$. For example, in giant molecular clouds cores of angular diameter 4 arcminutes - and with temperature $35 \mathrm{~K}$, $\mathrm{H}_{2}$ density $5 \times 10^{5} \mathrm{~cm}^{-3}$ and $\mathrm{H}_{2}$ column density $1.5 \times 10^{23} \mathrm{~cm}^{-2}$ - SWAS will detect $\mathrm{H}_{2} \mathrm{O}$ at $3 \sigma$ in 1 hour of integration provided its abundance relative to $\mathrm{H}_{2}$ exceeds $5 \times 10^{-9} . \mathrm{O}_{2}$ will be similarly detected provided its abundance relative to $\mathrm{H}_{2}$ exceeds $1.5 \times 10^{-6}$.

\section{Hot Shocked Regions}

Interstellar shock waves are a common phenomenon in regions of active star formation. When they propagate through molecular clouds, shock waves heat the gas, leading to substantial changes in the chemical composition.

\subsection{Theoretical Expectations}

Detailed theoretical modeling of molecular shock waves has been carried out by several groups. Slow, non-dissociative shocks have been considered, for example, by Elitzur and de Jong (1978), by Chernoff, Hollenbach and McKee (1982), and by Draine, Roberge and Dalgarno (1983), while fast dissociative shocks have been modeled by Hollenbach and McKee $(1979 ; 1989)$ and by Neufeld and Dalgarno (1989). Common to every one these calculations is the prediction that the water abundance in the hot region behind the shock will be substantially enhanced by chemical reactions with substantial activation energies that can be overcome only in the hot gas behind a shock wave. Detailed calculations show that behind a non-dissociative shock the $\mathrm{H}_{2} \mathrm{O}$ molecule accounts for most of the gas-phase oxygen that is not tied up in CO.

\subsection{Observations of MASER Emission}

Interstellar water masers are observed as intense spots of emission in the $22 \mathrm{GHz} 6_{25}-5_{23}$ radio line which have been widely detected within regions of active star formation. Large radial and/or proper motions suggest an association with hypersonic gas motions and thus with shock waves, and detailed calculations have shown (Elitzur, Hollenbach and McKee 1989) that the physical conditions within shocked dense clumps can be expected to produce maser emission with the enormous brightness temperatures $\left(>10^{12} \mathrm{~K}\right)$ 
that are typically observed. Thus it seems that in observing the $22 \mathrm{GHz}$ maser transition we are probing dense condensations within a shock-heated molecular outflow.

For twenty years following the first detection of the $22 \mathrm{GHz}$ maser transition (Cheung et al. 1969), no other masing transitions were unequivocally identified. The observational picture has been radically changed in the past two years, however, with the firm detection of maser action in three additional transitions: the $10_{29}-9_{36}$ line at $321 \mathrm{GHz}$ (Menten, Melnick and Phillips 1990); the $3_{13}-2_{20}$ line at $183 \mathrm{GHz}$ (Cernicharo et al. 1990); and the $5_{15}-4_{22}$ line at $325 \mathrm{GHz}$ (Menten et al. 1990). Maser action in each of these lines is expected under much the same physical conditions that are required to produce the $22 \mathrm{GHz}$ water maser (Neufeld and Melnick 1991), and the ratios of different maser lines can be used as a powerful constraint upon the nature of the emitting region. The $321 \mathrm{GHz} / 22 \mathrm{GHz}$ line ratio, in particular, is a valuable probe of the temperature (Neufeld and Melnick 1990), which indicates that in at least some masing regions the gas temperature must exceed $900 \mathrm{~K}$ : such high $\mathrm{H}_{2} \mathrm{O}$ temperatures can be achieved only behind non-dissociative (rather than dissociative) shocks.

\subsection{FutURE PROSPECTS}

While $\mathrm{H}_{2} \mathrm{O}$ maser line emissions can be used to probe dense condensations within a shocked molecular flow, non-masing far-infrared $\mathrm{H}_{2} \mathrm{O}$ emissions account for much of the energy radiated by the lower density material. Models for the emission spectrum of slow, non-dissociative shocks - such as the shocks believed to be present in the Orion-KL region (Draine and Roberge 1982) - have shown (Neufeld and Melnick 1987) that about one-half of the emitted radiation is expected to emerge in far-infrared rotational lines of $\mathrm{H}_{2} \mathrm{O}$. To date, the severity of the atmospheric attenuation has prevented any far-infrared $\mathrm{H}_{2} \mathrm{O}$ lines from being observed even at airplane altitudes. However, several hundred such lines are expected to show fluxes greater than $10^{-18} \mathrm{~W} \mathrm{~cm}^{-2}$ into a 1 arcminute beam and therefore to be readily detectable from space by the Infrared Space Observatory (ISO) - an ESA mission which is scheduled for launch in 1993 - and a few lines are predicted to show fluxes greater than $10^{-15} \mathrm{~W} \mathrm{~cm}^{-2}$ into a 1 arcminute beam! Within the next few years, the careful modeling of spectrophotometric observations of several far-infrared $\mathrm{H}_{2} \mathrm{O}$ lines promises to yield reliable estimates for the $\mathrm{H}_{2} \mathrm{O}$ abundance within hot shocked gas. Such observations will therefore provide a crucial test of one of the key untested predictions of gas-phase interstellar chemistry: that oxygen is efficiently incorporated into water within hot shock-heated regions of the interstellar medium. 


\section{Acknowledgements}

I am grateful for partial travel support from the American Astronomical Society and from an IAU Young Astronomer Grant.

\section{References}

Black, J. H., and Smith, P. L. 1984, ApJ, 277, 562.

Cernicharo, J., Thum, C., Hein, H., John, D., Garcia, P., and Mattioco, F. 1990, A\& A, 231, L15.

Chernoff, D. F., Hollenbach, D. J., and McKee, C. F. 1982, ApJL, 259, L97.

Cheung, A. C., Rank, D. M., Townes, C. H., Thornton, D. D., and Welch, W. J. 1969, Nature, 221, 626.

Draine, B. T., Roberge, W. G., and Dalgarno A. 1983, ApJ, 264, 485.

Draine, B. T., and Roberge, W. G. 1982, ApJL, 259, L91.

Elitzur, M., and de Jong, T. 1978, A\& A, 67, 323.

Elitzur, M., Hollenbach, D. J., and McKee, C. F. 1989, ApJ, 246, 983.

Goldsmith, P. F., and Langer, W. D. 1978, ApJ, 222, 881.

Goldsmith, P. F., Snell, R. L., Erickson, N. R., Dickman, R. L., Schloerb, F. P., and Irvine, W. M. 1985, ApJ, 289, 613.

Goldsmith, P. F., and Young, J. S. 1989, ApJ, 341, 718.

Herbst, E., and Leung, C. M. 1989, ApJS, 69, 271.

Hollenbach, D. J., and McKee, C. F. 1979, ApJS, 41, 555.

Hollenbach, D. J., and McKee, C. F. 1989, ApJ, 342, 306.

Langer, W. D., Graedel, T. E., Frerking, M. A., and Armentrout, P. B. 1984, ApJ, 277, 581.

Langer, W. D., and Graedel, T. E. 1989, ApJS, 69, 241.

Liszt, H. S. 1985, ApJ, 298, 281.

Liszt, H. S., and vandenBout, P. A. 1985, ApJ, 291, 178.

Melnick, G. J. et al. 1991, in Atoms, Ions and Molecules: New Results in Spectral Line Astrophysics, ed. A. D. Haschick and P. T. Ho (San Francisco: Astronomical Soc. of the Pacific), p. 439.

Menten, K. M., Melnick, G. J., and Phillips, T. G. 1990, ApJL, 350, L41.

Menten, K. M., Melnick, G. J., Phillips, T. G., and Neufeld, D. A. 1990, ApJL, 363, L27.

Neufeld, D. A., and Dalgarno A. 1989, ApJ, 340, 869.

Neufeld, D. A., and Melnick, G. J. 1987, ApJ, 322, 266.

Neufeld, D. A., and Melnick, G. J. 1990, ApJL, 352, L9.

Neufeld, D. A., and Melnick, G. J. 1991, ApJ, 368, 215.

Sternberg, A., Lepp, S., and Dalgarno, A. 1987, ApJ, 320, 676.

Wannier, P. G. et al. 1991, ApJ, 377, 171. 


\section{QUESTION AND ANSWER}

M.Guelin: (comment) Cernicharo et al. (1990) detected a broad smooth spectral component in the $183 \mathrm{GHzs}$ line profile toward W49, that they interpreted as thermal emission of $\mathrm{H}_{2} \mathrm{O}$. Alternately, the broad line wings could be a large collection of maser features. If the line wings are thermal and optically thin, the water abundance derived is $\sim 10^{-5}$ of $\mathrm{H}_{2}$ and is consistent with that deduced from $\mathrm{H}_{2}^{18} \mathrm{O}$ and HDO observaations (Jacq et al. $1990)$. 